\title{
GROMOV-WITTEN INVARIANTS OF SYMPLECTIC SUMS
}

\author{
Eleny-Nicoleta Ionel and Thomas H. Parker
}

The natural sum operation for symplectic manifolds is defined by gluing along codimension two submanifolds. Specifically, let $X$ be a symplectic $2 n$-manifold with a symplectic $(2 n-2)$-submanifold $V$. Given a similar pair $(Y, \bar{V})$ with a symplectic identification $V=\bar{V}$ and a complex anti-linear isomorphism between the normal bundles of $V$ and $\bar{V}$, we can form the symplectic sum $Z=X \#_{V=\bar{V}} Y$. This note announces a general formula for computing the Gromov-Witten invariants of the sum $Z$ in terms of relative Gromov-Witten invariants of $(X, V)$ and $(Y, \bar{V})$.

Section 1 is a review of the GW invariants for symplectic manifolds and the associated invariants, which we call $T W$ invariants, that count reducible curves. The corresponding relative invariants of a symplectic pair $(X, V)$ are defined in section 2. The sum formula is stated in a special case in section 3 , and in general as Theorem 4.1. The last section presents two applications: a short derivation of the Caporaso-Harris formula $[\mathrm{CH}]$, and new proof that the rational enumerative invariants of the rational elliptic surface are given by the "modular form" (5.3).

Related results, involving symplectic sums along contact manifolds, are being developed by Li and Ruan [LR] and by Eliashberg and Hofer.

\section{Symplectic invariants}

The moduli space of $(J, \nu)$-holomorphic maps from genus $g$ curves with $n$ marked points representing a class $A$ in the free part of $H_{2}(X ; \mathbb{Z})$ has a compactification $\overline{\mathcal{M}}_{g, n}(X, A)$. This comes with a map

$$
\overline{\mathcal{M}}_{g, n}(X, A) \longrightarrow \overline{\mathcal{M}}_{g, n} \times X^{n} \times H_{2}^{\text {free }}(X ; \mathbb{Z}),
$$

where the first factor is the "stabilization" map st to the Deligne-Mumford moduli space (defined by collapsing all unstable components of the domain curve), the second factor records the images of the marked points, and the last one keeps track of the homology class $A$ of the image. After perturbation (cf. [LT]), the image defines a rational homology class,

$$
\left[\overline{\mathcal{M}}_{g, n}(X, A)\right] \in H_{*}\left(\overline{\mathcal{M}}_{g, n} ; \mathbb{Q}\right) \otimes H_{*}\left(X^{n} ; \mathbb{Q}\right) \otimes H_{*}\left(H_{2}^{\text {free }}(X ; \mathbb{Z}) ; \mathbb{Q}\right)
$$

for each choice $g, n, A$. The last term in (1.2) can be identified with the rational group ring $R H_{2}(X)$ of $H_{2}^{\text {free }}(X ; \mathbb{Z})$, that is, with finite sums $\sum c_{A} t_{A}$ over $A \in$ $H_{2}^{\text {free }}(X ; \mathbb{Z})$ where $c_{A} \in \mathbb{Q}$ and the $t_{A}$ are variables satisfying $t_{A} t_{B}=t_{A+B}$.

Received June 2, 1998.

Both authors are partially supported by the National Science Foundation. 
After summing on $A$ and dualizing, (1.2) defines a map

$$
G W_{g, n, X}: H^{*}\left(\overline{\mathcal{M}}_{g, n}\right) \otimes H^{*}\left(X^{n}\right) \rightarrow R H_{2}(X) .
$$

(This is well-defined for $2 g+n \geq 3$, and we will always assume that it has been extended to all $g, n$ by the stabilization procedure of [IP1] $\S 3)$.

Finally, we sum over $n$ and $g$ by setting $\overline{\mathcal{M}}=\bigcup_{g, n} \overline{\mathcal{M}}_{g, n}$, letting $\mathbb{T}(X)$ denote the total tensor algebra $\mathbb{T}\left(H^{*}(X)\right)$ on the rational cohomology of $X$, and introducing a variable $\lambda$ to keep track of the euler class. The total Gromov-Witten invariant is then the map

$$
G W_{X}: H^{*}(\overline{\mathcal{M}}) \otimes \mathbb{T}(X) \rightarrow R H_{2}(X)[\lambda],
$$

which is linear in the second factor and defined by the Laurent expansion

$$
G W_{X}=\sum_{g, n, A} \frac{1}{n !} G W_{g, n, A, X} t_{A} \lambda^{2 g-2} .
$$

The diagonal action of the symmetric group on $\overline{\mathcal{M}}_{g, n} \times X^{n}$ leaves $G W_{X}$ invariant up to sign, and if $\kappa \in H^{*}\left(\overline{\mathcal{M}}_{g, n}\right)$ then $G W_{X}(\kappa, \alpha)$ vanishes unless $\alpha$ is a tensor of length $n$.

We can recover the familiar geometric interpretation of these invariants by evaluating on cohomology classes. Given $\kappa \in H^{*}(\overline{\mathcal{M}} ; \mathbb{Q})$ and a vector $\alpha=$ $\left(\alpha_{1}, \ldots, \alpha_{n}\right)$ of rational cohomology classes in $X$ of length $|\alpha|=n$, fix a generic $(J, \nu)$ and generic geometric representatives $K$ and $A_{i}$ of the Poincaré duals of $\kappa$ and of the $\alpha_{i}$. Then $G W_{g, n, A, X}(\kappa, \alpha)$ counts (with orientation) the number of genus $g(J, \nu)$-holomorphic maps $f: C \rightarrow X$ with $C \in K$ and $f\left(x_{i}\right) \in A_{i}$ for each marked point $x_{i}$. By the usual dimension counts, this vanishes unless

$$
\operatorname{deg} \kappa+\sum \operatorname{deg} \alpha_{i}-2|\alpha|=(\operatorname{dim} X-6)(1-g)-2 K_{X} \cdot A,
$$

where $K_{X}$ is the canonical class of $X$.

It is sometimes useful to also incorporate the ' $\psi$ classes'. There are canonical oriented real 2-plane bundles $\mathcal{L}_{i}$ over $\overline{\mathcal{M}}_{g, n}(X, A)$ whose fiber at each map $f$ is the cotangent space to the domain curve at the $i^{\text {th }}$ marked point. Let $\psi_{i}$ be the euler class of $\mathcal{L}_{i}$, and for each vector $D=\left(d_{1}, \ldots d_{n}\right)$ of non-negative integers let $\psi_{D}=\psi_{1}^{d_{1}} \cup \cdots \cup \psi_{n}^{d_{n}}$. Replacing the lefthand side of (1.2) by the pushforward of the cap product $\psi_{D} \cap\left[\overline{\mathcal{M}}_{g, n}(X, A)\right]$ and again dualizing gives invariants

$$
G W_{g, n, D, X}: H^{*}\left(\overline{\mathcal{M}}_{g, n}\right) \otimes H^{*}\left(X^{n}\right) \rightarrow R H_{2}(X),
$$

which agree with (1.3) when $D$ is the zero vector. These invariants can be included in (1.4); the series (1.5) then has additional variables that keep track of the vector $D$. To keep the notation manageable we will not do this explicitly, although these invariants will reappear at the end of section 4. (The similar classes $\phi_{i}$ defined by the cotangent space of the stabilized domain are already part of (1.4)). 
The invariant (1.4) counts $(J, \nu)$-holomorphic maps from connected domains. It is often more natural to work with domains with more components, as Taubes does in [T] (see also [IP1]). Let $\widetilde{\mathcal{M}}_{\chi, n}$ be the space of all stable curves of euler characteristic $\chi$ with any number of components, of any possible genus, and with $n$ ordered points distributed in all possible ways. More precisely, let $\mathcal{P}_{n}$ be the set of all ordered partitions of the set $\left\{x_{1}, \ldots x_{n}\right\}$. Each $\pi=\left(\pi_{1}, \ldots, \pi_{\ell}\right) \in \mathcal{P}_{n}$ records which marked points go on each component. Then

$$
\widetilde{\mathcal{M}}_{\chi, n}=\bigsqcup_{\pi \in \mathcal{P}_{n}} \bigsqcup_{g_{i}} \overline{\mathcal{M}}_{g_{1}, \pi_{1}} \times \ldots \times \overline{\mathcal{M}}_{g_{l}, \pi_{l}},
$$

where $\overline{\mathcal{M}}_{g_{i}, \pi_{i}}$ is the Deligne-Mumford space with $n_{i}$ marked points labeled by the set $\pi_{i}$ and where the second sum is over all $g_{i}$ with $\sum\left(2-2 g_{i}\right)=\chi$. Define the "Taubes-Witten" invariant

$$
T W_{X}: H^{*}(\widetilde{\mathcal{M}}) \otimes \mathbb{T}(X) \rightarrow R H_{2}(X)[\lambda],
$$

by

$$
T W_{X}=e^{G W_{X}} .
$$

As before, when this is expanded as a Laurent series

$$
T W_{X}(\kappa, \alpha)=\sum \frac{1}{n !} T W_{X, A, \chi, n}(\kappa, \alpha) \lambda^{-\chi} t_{A},
$$

the coefficients count the number of curves (not necessarily connected) with euler characteristic $\chi$ representing $A$ and satisfying the constraints $(\kappa, \alpha)$.

Remark 1.1 Specifically, if $\alpha=\alpha_{1} \otimes \cdots \otimes \alpha_{n}$, then for each partition $\pi=$ $\left(\pi_{1}, \ldots \pi_{\ell}\right)$ let $\alpha_{\pi_{i}}$ be the product of $\alpha_{j}$ for all $j \in \pi_{i}$. If $\kappa=\kappa_{1} \otimes \cdots \otimes \kappa_{\ell}$, then:

$$
\begin{aligned}
T W_{X, n}(\kappa, \alpha)= & \\
& \sum_{\pi \in \mathcal{P}_{n}} \frac{\varepsilon(\pi)}{\ell !}\left(\begin{array}{c}
n \\
n_{1}, \ldots, n_{\ell}
\end{array}\right) G W_{X, n_{1}}\left(\kappa_{1}, \alpha_{\pi_{1}}\right) \otimes \cdots \otimes G W_{X, n_{\ell}}\left(\kappa_{\ell}, \alpha_{\pi_{\ell}}\right),
\end{aligned}
$$

where $\varepsilon_{\pi}= \pm 1$ depending on the sign of the permutation $\left(\pi_{1}, \ldots, \pi_{\ell}\right)$ and the degrees of $\alpha$. Note that $A$ and $\chi$ add when one take disjoint unions, so the variables $t_{A}$ and $\lambda$ multiply.

\section{Relative invariants}

The invariants of Section 1 can be extended to invariants for $(X, \omega)$ relative to a codimension two symplectic submanifold $V$ (a related invariant has been defined by Li-Ruan [LR]). This requires addressing two major issues: "rim tori" and higher order contact between the holomorphic curves and $V$.

Each almost complex structure $J$ on $X$ compatible with the symplectic form determines a metric by $g(v, w)=\omega(v, J w)$, a Levi-Civita connection $\nabla$, and a splitting of $T X$ along $V$ as the sum $T V \oplus N_{V}$ of tangent and normal bundles. Let $\mathcal{J}^{V}$ be the space of pairs $(J, \nu)$ where $\nu$ is a perturbation (as in [RT]) satisfying the following three conditions on the 1-jet of $(J, \nu)$ along $V$ : (i) $J$ preserves $T V$ 
and $\left.\nu\right|_{V}$ takes values in $T V$, (ii) the bundle map $A: T X \otimes T X \rightarrow N_{V}$ defined by $A(\xi, v)=\left(\nabla_{\xi} J+J \nabla_{J \xi} J\right)^{N}(v)$ satisfies $A(\xi, v)=A(v, \xi)$ for all $\xi \in N_{V}$ and $v \in T V$, and (iii) for each $\xi \in N_{V},\left(\nabla_{\xi} \nu+J \nabla_{J \xi} \nu-J \nabla_{\nu()} J\right)^{N} \in \Omega^{0,1}\left(N_{V}\right)$ vanishes. (These conditions ensure that for each $(J, \nu)$-holomorphic map whose image $C$ lies entirely in $V$, the restriction of the linearization $D_{C}$ to the normal bundle to $V$ is a complex operator.) A $(J, \nu)$-holomorphic map into $X$ is $V$ regular if the inverse image of $V$ is a finite set of points. For each $(g, n)$, the $V$-regular maps form an open subset $\mathcal{M}_{g, n}^{V}(X)$ of the moduli space of maps, and this is a manifold for generic $(J, \nu) \in \mathcal{J}^{V}$. We will write this moduli space as a disjoint union of strata and compactify each strata separately.

The strata are labeled by sequences $s=\left(s_{1}, s_{2}, \ldots, s_{\ell}\right)$ of integers with $s_{i} \geq 1$. Let $\mathcal{S}$ be the set of all such sequences and define the degree, length, and order of $s \in \mathcal{S}$ by

$$
\operatorname{deg} s=\sum s_{i} \quad \ell(s)=\ell, \quad|s|=s_{1} s_{2} \cdots s_{\ell} .
$$

Associated to each $s$ is the moduli space

$$
\mathcal{M}_{g, n, s}^{V}(X) \subset \mathcal{M}_{g, n+\ell(s)}^{V}(X),
$$

consisting of those maps $f$ such that $f^{-1}(V)$ is exactly the points $x_{i}, n+1 \leq$ $i \leq n+\ell(s)$, each with multiplicity $s_{i}$. Then $\mathcal{M}_{g, n, s}^{V}(X)$ defines a stratum of $\mathcal{M}_{g, n}^{V}(X)$ via the projection that forgets the last $\ell(s)$ points, and $\mathcal{M}_{g, n}^{V}(X)$ is the disjoint union of these strata.

To compactify $\mathcal{M}_{g, n, s}^{V}(X)$, we introduce the "symplectic compactification" $\widehat{X}$ of $X \backslash V$. This is a compact, symplectic manifold whose boundary is the circle bundle $S$ of the normal bundle to $V$ in $X$ together with a projection $\pi: \widehat{X} \rightarrow X$ which is the projection $S \rightarrow V$ on the boundary and is a symplectomorphism in the interior. Let $V^{*}$ be $V$ with the discrete topology, and let $S^{*}$ be $S$ topologized as the disjoint union of its fiber circles. Then $\pi: S^{*} \rightarrow V^{*}$ and the inclusions $V^{*} \subset X$ and $S^{*} \subset \hat{X}$ are continuous. The long exact sequence of the pair $\left(\hat{X}, S^{*}\right)$ gives maps

$$
H_{2}(\hat{X}) \longrightarrow H_{2}\left(\hat{X}, S^{*}\right) \stackrel{\rho}{\longrightarrow} H_{1}\left(S^{*}\right) .
$$

Each $V$-regular map lifts to a map into $\hat{X}$; this determines an element of $H_{2}\left(\hat{X}, S^{*}\right)$ that projects under $\rho$ to an element of the symmetric product $\operatorname{Sym} V$. Similarly, each $f \in \mathcal{M}_{g, n, s}^{V}(X)$ lifts to $\hat{X}$, and evaluation at the last $\ell(s)$ marked points gives a map into the space

$$
\mathcal{S} V=\bigcup_{s \in \mathcal{S}} V_{s}
$$

where each $V_{s}$ is a copy of $V^{\ell(s)}$. We give $S V$ the topology of that makes this a disjoint union. Restricting (2.1) to Sym $V$ and pulling back by the projection $\mathcal{S} V \rightarrow \operatorname{Sym} V$, then gives a space $\mathcal{H}_{X}^{V}=H_{2}\left(\hat{X}, S^{*}\right){ }{ }_{\text {Sym } V} \mathcal{S} V$ with an 
evaluation map $\varepsilon$ into $\mathcal{S} V$ :

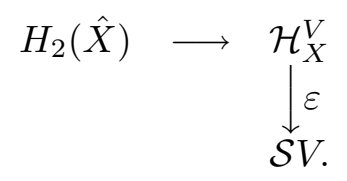

We topologize $\mathcal{H}_{X}^{V}$ so that $\varepsilon$ is a covering map. With this setup, lifting maps into $\hat{X}$ gives a diagram

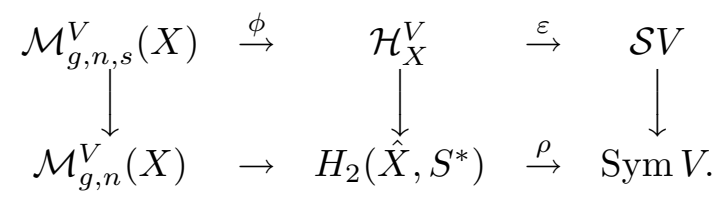

Note that the intersection divisor with $V$ can be recovered by applying $\rho$, and that the image of $\phi$ implicitly describes that class of the lift in $H_{2}(\hat{X}, S)$. However, these data cannot be separated because the covering (2.2) is not, in general, trivial.

Remark 2.1 The covering (2.2) can be partially trivialized. Let $\mathcal{R}_{X}$ be the kernel of the projection $H_{2}(\hat{X}) \rightarrow H_{2}(X)$. Elements of $\mathcal{R}_{X}$ are called rim tori because they can be represented by tori of the form $\alpha \times \mu$ where $\alpha$ is a curve in $S$ and $\mu$ is a fiber of $S \rightarrow V$. Projecting 2-cycles in $\left(\hat{X}, S^{*}\right)$ into $X$ defines a map $\pi_{*}: \mathcal{H}_{X}^{V} \rightarrow H_{2}(X)$. Then (2.2) can be rewritten as a covering map

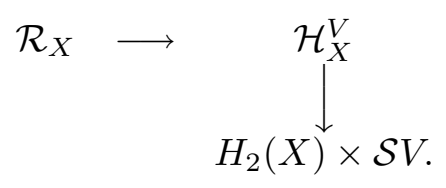

Lemma 2.2. For generic $(J, \nu)$ in $\mathcal{J}^{V}$, the image of $\mathcal{M}_{g, n, s}^{V}(X)$ under $\phi$ has compact closure in $\mathcal{H}_{X}^{V}$ for each $s \in \mathcal{S}$, and these images define elements of $H_{*}\left(\mathcal{H}_{X}^{V} ; \mathbb{Q}\right)$.

The relative GW invariant of the pair $(X, V)$ is then obtained by repeating the discussion leading from (1.2) to (1.4). Writing $\mathbb{T}\left(H^{*}(X, V)\right)$ as $\mathbb{T}(X, V)$, the relative invariant is thus a map

$$
G W_{X}^{V}: H^{*}(\overline{\mathcal{M}}) \otimes \mathbb{T}(X, V) \longrightarrow H_{*}\left(\mathcal{H}_{X}^{V}\right)[\lambda],
$$

with an expansion

$$
G W_{X}^{V}=\sum_{g, n} \frac{1}{n !} G W_{g, n, X}^{V} \lambda^{2 g-2} .
$$

Formula (1.7) extends this to a relative Taubes-Witten invariant

$$
T W_{X}^{V}: H^{*}(\overline{\mathcal{M}}) \otimes \mathbb{T}(X, V) \rightarrow H_{*}\left(\mathcal{H}_{X}^{V}\right)[\lambda] .
$$


When $V$ is the empty set $\mathcal{H}_{X}^{V}$ is $H_{2}(X)$ and the relative invariant takes values in $R H_{2}(X)$. The relative and absolute invariants are then equal: $G W_{X}^{\emptyset}=G W_{X}$.

These relative invariants depend only on the symplectic structure:

Theorem 2.3. $G W^{V}$ and $T W^{V}$ are independent of the generic $(J, \nu)$ in $\mathcal{J}^{V}$.

The geometric interpretation of the relative invariant (2.4) is similar to that of (1.5). Given $\kappa \in H^{*}(\overline{\mathcal{M}})$, a vector $\alpha=\left(\alpha_{1}, \ldots, \alpha_{n}\right)$ of classes in $H^{*}(X, V)$, and a $\gamma \in H^{*}\left(\mathcal{H}_{X}^{V}\right)$, fix generic geometric representatives $K \subset \overline{\mathcal{M}}_{g, n}, \Gamma \subset \mathcal{H}_{X}^{V}$ and $A_{i} \subset X$ of their Poincaré duals. Then the evaluation pairing $\gamma \cdot G W_{g, X}^{V}(\kappa, \alpha)$ counts the oriented number of genus $g(J, \nu)$-holomorphic maps $f: C \rightarrow X$ with $C \in K, \phi(f) \in \Gamma$, and $f\left(x_{i}\right) \in A_{i}$ for each marked point $x_{i}$.

Note that the condition $\phi(f) \in \Gamma$ constrains both the homology class $A$ of the map and the boundary values of the curve. In the special case when there are no rim tori, these homology and the boundary value constraints can be fully separated.

Example 2.4 When there are no rim tori, $\mathcal{H}_{X}^{V}$ is the subset of $H_{2}(X) \times \bigcup_{s} V^{\ell(s)}$ consisting of pairs $(A, s)$ with $\operatorname{deg} s=A \cdot V$. The homology of $\mathcal{H}_{X}^{V}$ is the corresponding subalgebra of $R H_{2}(X) \otimes \mathbb{C T}$ where $\mathbb{C T}$ is the "contact tensor algebra" of $V$ :

$$
\mathbb{C} \mathbb{T}(V)=\mathbb{T}\left(H_{*}(V) \times \mathbb{N}\right) .
$$

The relative invariants are then maps

$$
H^{*}(\overline{\mathcal{M}}) \otimes \mathbb{T}(X, V) \rightarrow \mathbb{C} \mathbb{T}(V) \otimes R H_{2}(X)[\lambda],
$$

and have Laurent expansions like (1.5) with coefficients in $\mathbb{C} \mathbb{T}(V)$. In fact, a basis for the contact algebra is given by elements of the form $C_{s, \gamma}=C_{s_{1}, \gamma_{1}} \otimes \cdots \otimes C_{s_{\ell}, \gamma_{\ell}}$, where $\gamma_{i}$ are elements of $H_{*}(V ; \mathbb{Q})$ and $s_{i} \geq 1$ are integers. Let $\left\{C_{s, \gamma}^{*}\right\}$ denote the dual basis. With $\kappa$ and $\alpha$ as above, we can expand

$$
\sum_{s, \gamma} G W_{g, A, X}^{V}\left(\kappa, \alpha ; C_{s, \gamma}\right) C_{s, \gamma}^{*} t_{A} \lambda^{2 g-2},
$$

where the coefficients count the oriented number of genus $g(J, \nu)$-holomorphic maps $f: C \rightarrow X$ with $C \in K, f\left(x_{i}\right) \in A_{i}$, and having a contact of order $s_{j}$ with $V$ along fixed representatives $\Gamma_{j}$ of the Poincaré duals of the $\gamma_{j}$.

\section{The convolution}

Having defined the relative invariants, we now turn to the problem of describing how they behave under the symplectic sum operation. The goal is to give a formula expressing the absolute invariants of a symplectic sum $Z=X_{V=\bar{V}} Y$ in terms of the relative invariants of $X$ and $Y$. This turns out to be most natural when one works with the $T W$ invariants, and when one works with relative invariants throughout.

Let $X$ be a symplectic manifold with two disjoint symplectic submanifolds $U$ and $V$ with real codimension two. Suppose that $V$ is symplectically identified 
with a submanifold of similar triple $(Y, \bar{V}, W)$ and that the normal bundles of $V \subset X$ and $\bar{V} \subset Y$ have opposite chern classes. Let $(Z, U, W)$ be the resulting symplectic sum. We will choose $(J, \nu)$ on the $X$ and $Y$ sides so that the 1 -jets of $(J, \nu)$ match along $V=\bar{V}$. In this section we will define a "convolution" operation and establish a formula of the form

$$
T W_{X}^{U \cup V} * T W_{Y}^{\bar{V} \cup W}=T W_{Z}^{U \cup W},
$$

under the assumption that all curves contributing to the invariants are $V$-regular (this condition will be eliminated in the next section).

The convolution operation is assembled from several pieces. The first ingredient is a gluing map defined on Deligne-Mumford spaces. Given stable curves $C_{1}$ and $C_{2}$ (not necessarily connected) with euler characteristics $\chi_{i}$ and $n_{i}+\ell$ marked points, we can construct a new curve by identifying the last $\ell$ marked points of $C_{1}$ with the last $\ell$ marked points of $C_{2}$, and then forgetting the marking of these new double points. This defines an attaching map

$$
\xi_{\ell}: \widetilde{\mathcal{M}}_{\chi_{1}, n_{1}+\ell} \times \widetilde{\mathcal{M}}_{\chi_{2}, n_{2}+\ell} \longrightarrow \widetilde{\mathcal{M}}_{\chi_{1}+\chi_{2}-2 \ell, n_{1}+n_{2}}
$$

whose image is a subvariety of complex codimension $\ell$. Taking the union over all $\chi_{1}, \chi_{2}, n_{1}$ and $n_{2}$ gives a map $\xi_{\ell}: \widetilde{\mathcal{M}} \times \widetilde{\mathcal{M}} \rightarrow \widetilde{\mathcal{M}}$ for each $\ell$.

The second ingredient describes how maps are glued along $V$. Consider the evaluation map

$$
\mathrm{ev}: \mathcal{M}^{V}(X) \times \mathcal{M}^{\bar{V}}(Y) \stackrel{\phi}{\longrightarrow} \mathcal{H}_{X}^{V} \times \mathcal{H}_{Y}^{\bar{V}} \stackrel{\varepsilon}{\longrightarrow} \mathcal{S} V \times \mathcal{S} V
$$

defined by the top row of (2.3) on each factor; this evaluation map records the intersection points with $V$, together with their multiplicities. The diagonal of $\mathcal{S} V \times \mathcal{S} V$ is the union of components

$$
\Delta_{s} \subset \mathcal{S} V \times \mathcal{S} V
$$

labeled by sequences $s$. With this notation, we can state our first main result. It is a 'gluing theorem' for families of pseudo-holomorphic maps which is proved by PDE methods [IP2].

Theorem 3.1. For each sequence $s$, there is an $\frac{|s|}{\ell(s) !}$-fold cover of $e v^{-1}\left(\Delta_{s}\right)$, denoted $\mathcal{M}^{V}(X) \times_{e v_{s}} \mathcal{M}^{\bar{V}}(Y)$, and a commutative diagram

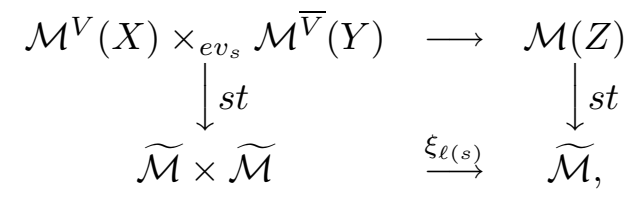

where the top arrow is a diffeomorphism. 
The next step is to pass to homology. We can glue a curve in $X$ to a curve in $Y$ provided the two curves meet $V$ at the same points with the same multiplicity; this defines a map

$$
g: \mathcal{H}_{X}^{U, V} \times_{\varepsilon} \mathcal{H}_{Y}^{\bar{V}, W} \rightarrow \mathcal{H}_{Z}^{U, W} .
$$

Combining this with the map $\xi_{\ell}$ above gives the convolution operator that describes how homology classes of maps combine in the gluing operation for the symplectic sum.

Definition 3.2. The convolution operator

$$
*: H_{*}\left(\widetilde{\mathcal{M}} \times \mathcal{H}_{X}^{U, V} ; \mathbb{Q}[\lambda]\right) \otimes H_{*}\left(\widetilde{\mathcal{M}} \times \mathcal{H}_{Y}^{\bar{V}, W} ; \mathbb{Q}[\lambda]\right) \longrightarrow H_{*}\left(\widetilde{\mathcal{M}} \times \mathcal{H}_{Z}^{U, W} ; \mathbb{Q}[\lambda]\right),
$$

is given by

$$
\begin{aligned}
(\kappa \otimes h) * & \left(\kappa^{\prime} \otimes h^{\prime}\right)= \\
& \sum_{s} \frac{|s|}{\ell(s) !}\left(\xi_{\ell(s)}\right)_{*}\left(\kappa \otimes \kappa^{\prime}\right) g_{*}\left[h \times\left. h^{\prime}\right|_{\varepsilon^{-1}\left(\Delta_{s}\right)}\right] \lambda^{2 \ell(s)} .
\end{aligned}
$$

To conform with this definition, we will regard the $T W$ invariant of $X$ as a map

$$
T W_{X}^{U, V}: \mathbb{T}(X, V) \rightarrow H_{*}\left(\widetilde{\mathcal{M}} \times \mathcal{H}_{Z}^{U, V} ; \mathbb{Q}[\lambda]\right),
$$

by dualizing the first term in (2.5). Finally, a constraint $\alpha \in H^{*}(Z)$ separates as $\alpha_{X}+\alpha_{Y}$ if its Poincaré dual $P D(\alpha)$ is the image of $\left(P D\left(\alpha_{X}\right), P D\left(\alpha_{Y}\right)\right)$ under the Mayer-Vietoris map $H_{*}(X \backslash V) \oplus H_{*}(Y \backslash \bar{V}) \rightarrow H_{*}(Z)$. These generate a subalgebra $\mathbb{T}_{s}(Z)$ of $\mathbb{T}(Z)$, namely the span of tensors of the form

$$
\alpha=\alpha_{X}+\alpha_{Y}
$$

with $\alpha_{X} \in \mathbb{T}(X, V)$ and $\alpha_{Y} \in \mathbb{T}(Y, \bar{V})$. Then, when $\alpha$ has this form and assuming that all curves contributing to the invariants are $V$-regular, (3.1) holds in the sense that

$$
T W_{Z}^{U, W}(\alpha)=T W_{X}^{U, V}\left(\alpha_{X}\right) * T W_{Y}^{\bar{V}, W}\left(\alpha_{Y}\right) .
$$

Example 3.3 (The S-matrix) Starting from the normal bundle $N_{V}$ of $V$ in $X$, we can form the $\mathbb{P}^{1}$ bundle $\mathbb{F}=\mathbb{P}\left(N_{V} \oplus \mathbb{C}\right)$ over $V$. In $\mathbb{F}$, the zero section $V$ and the infinity section $\bar{V}$ are disjoint symplectic submanifolds. The symplectic sum of $(X, U, V)$ and $(\mathbb{F}, \bar{V}, V)$ is a symplectic deformation of $(X, U, V)$, so has the same $T W$ invariant. The convolution then defines a operation

$$
H_{*}\left(\widetilde{\mathcal{M}} \times \mathcal{H}_{X}^{U, V} ; \mathbb{Q}[\lambda]\right) \otimes H_{*}\left(\widetilde{\mathcal{M}} \times \mathcal{H}_{\mathbb{F}}^{\bar{V}, V} ; \mathbb{Q}[\lambda]\right) \longrightarrow H_{*}\left(\widetilde{\mathcal{M}} \times \mathcal{H}_{X}^{U, V} ; \mathbb{Q}[\lambda]\right) .
$$

Thus for each choice of constraints $\alpha \in \mathbb{T}(\mathbb{F}, V \cup \bar{V})$, the $T W$ invariant of $\mathbb{F}$ relative to its zero and infinity section defines an "S-matrix"

$$
T W_{\mathbb{F}}^{\bar{V}, V}(\alpha) \in \operatorname{End}\left(H_{*}\left(\widetilde{\mathcal{M}} \times \mathcal{H}_{X}^{U, V} ; \mathbb{Q}[\lambda]\right)\right),
$$


which describes how families of curves on $X$ are modified - "scattered" - as they pass through a neck modeled on $(\mathbb{F}, \bar{V}, V)$ containing the constraints $\alpha$.

The identity in the endomorphism algebra (3.6) is always realized as the convolution by the element

$$
\mathbb{I} \in H_{*}\left(\widetilde{\mathcal{M}} \times \mathcal{H}_{\mathbb{F}}^{\bar{V}, V} ; \mathbb{Q}[\lambda]\right),
$$

corresponding to that part of $T W(1)$ coming from the maps whose domain is unstable and whose image is a multiple cover of a fiber of $\mathbb{F}$. As in physics, it is convenient to write the $S$-matrix (3.6) as $\mathbb{I}+R^{\bar{V}, V}$; the inverse of the $S$-matrix is then given by the series

$$
\begin{aligned}
\left(T W_{\mathbb{F}}^{\bar{V}, V}\right)^{-1} & = \\
& \mathbb{I}-R^{\bar{V}, V}+R^{\bar{V}, V} * R^{\bar{V}, V}-R^{\bar{V}, V} * R^{\bar{V}, V} * R^{\bar{V}, V}+\ldots
\end{aligned}
$$

Example 3.4 When $V=\mathbb{P}^{1}, \mathbb{F} \rightarrow V$ is one of the rational ruled surfaces with its standard symplectic and holomorphic structure. If we wish to count all pseudo-holomorphic maps, without constraints on the genus or the induced complex structure, the relevant $\mathrm{S}$-matrix is the relative $T W$ invariant with $(\kappa, \alpha)=(1,1) \in H^{0}(\overline{\mathcal{M}}) \times H^{0}(X)$. This case works out neatly: a dimension count shows that $T W_{\mathbb{F}}^{\bar{V}}, V(1,1)=\mathbb{I}$.

\section{The sum formula}

The full formula for the invariants of a symplectic sum is obtained from (3.5) by a 'stretching the neck' argument. The sum $X \#_{V} Y$ can be viewed as assembled from $n+1$ symplectic sums, where the middle $n$ pieces are copies of the ruled space $\mathbb{F}$ associated to $V$. We again pick $(J, \nu)$ on the pieces of this sum so that their 1-jets match along each cut. The pigeon-hole principle implies that, for each $A \in H_{2}(Z)$, we can take $n$ sufficiently large so that all pseudo-holomorphic $A$-curves in $Z$ limit to $V$-regular curves in the $n+1$-fold sum. Thus the curves in $Z$ decompose into curves in $X$ joined to curves in $Y$ by a chain of curves in the intermediate copies of $\mathbb{F}$. Applying formula (3.5) along each cut yields the general sum formula.

Theorem 4.1. Let $(Z, U, W)$ be the symplectic sum of $(X, U, V)$ and $(Y, \bar{V}, W)$ along $V=\bar{V}$. Suppose that $\alpha \in \mathbb{T}(Z)$ separates as in (3.4). Then the relative $T W$ invariant of $Z$ is given in terms of the invariants of $(X, U, V)$ and $(Y, \bar{V}, W)$ by

$$
T W_{Z}^{U, W}(\alpha)=T W_{X}^{U, V}\left(\alpha_{X}\right) *\left(T W_{\mathbb{F}}^{\bar{V}, V}\right)^{-1} * T W_{Y}^{\bar{V}, W}\left(\alpha_{Y}\right),
$$

where the middle term is the inverse of the $S$-matrix (3.7).

One interesting special case is when $U$ and $W$ are empty and we put no constraints on the complex structure of the curves. Theorem 4.1 then gives a 
formula expressing the absolute invariant of $Z$ in terms of the relative invariants of $X$ and $Y$.

In this case the algebra of the formula simplifies: we drop all references to $\widetilde{\mathcal{M}}$ and, because $U$ and $W$ are empty, $H_{*}\left(\mathcal{H}_{Z}^{U, W}\right)$ is simply $R H_{2}(Z)$. The convolution (3.4) can then be thought of as a bilinear pairing

$$
\langle,\rangle: H_{*}\left(\mathcal{H}_{X}^{V} ; \mathbb{Q}[\lambda]\right) \otimes H_{*}\left(\mathcal{H}_{Y}^{\bar{V}} ; \mathbb{Q}[\lambda]\right) \longrightarrow R H_{2}(Z)[\lambda]
$$

In this case $g: \mathcal{H}_{X}^{V} \times_{\varepsilon} \mathcal{H}_{Y}^{\bar{V}} \rightarrow R H_{2}(Z)$, so for each sequence $s, \varepsilon^{-1}\left(\Delta_{s}\right)$ is the union of components $\Delta_{A, s}=\varepsilon^{-1}\left(\Delta_{s}\right) \cap g^{-1}(A)$, and the formula for the bilinear pairing becomes

$$
\left\langle h, h^{\prime}\right\rangle=\sum_{s} \frac{|s|}{\ell(s) !} \sum_{A \in H_{2}(Z)} g_{*}\left[\Delta_{A, s} \cap\left(h \times h^{\prime}\right)\right] t_{A} \lambda^{2 \ell(s)} .
$$

The $S$-matrix is the operator obtained by letting $T W_{\mathbb{F}}^{\bar{V}}, V(1,1) \in H_{*}\left(\mathcal{H}_{X}^{U, V} ; \mathbb{Q}[\lambda]\right)$ act by convolution; let $S_{\bar{V}, V}$ denote its inverse.

Corollary 4.2. Let $Z$ be the symplectic sum of $(X, V)$ and $(Y, \bar{V})$ and suppose that $\alpha \in \mathbb{T}(Z)$ separates as in (3.4). Then

$$
T W_{Z}(\alpha)=\left\langle T W_{X}^{V}\left(\alpha_{X}\right), S_{\bar{V}, V} \cdot T W_{Y}^{\bar{V}}\left(\alpha_{Y}\right)\right\rangle .
$$

Another simplification occurs when there are no rim tori in $X$ and $Y$, and therefore in $Z$. Then the relative invariants have an expansion of the form (2.6). For simplicity we take $U$ and $W$ both empty. Then $\mathcal{H}_{Z}^{U, W}$ is simply $H_{2}(Z)$ and the map $g$ of (3.2) is the identification $\mathcal{S} V \times{ }_{\rho} \mathcal{S} V$ with $\mathcal{S} V$ together with the addition $H_{2}(X) \times H_{2}(Y) \rightarrow H_{2}(Z)$. The key part of the convolution (3.4) is then given by the cap product with the Poincaré dual of the diagonal:

$$
g_{*}\left[h \times\left. h^{\prime}\right|_{\Delta_{s}}\right]=\sum_{B \in H_{2}(X)} \sum_{C \in H_{2}(Y)} \operatorname{PD}\left(\Delta_{s}\right) \cap\left(h \times h^{\prime}\right) t_{B} t_{C} .
$$

We can then 'split the diagonal' by fixing a basis $\left\{h^{p}\right\}$ of $H^{*}(\mathcal{S} V)$ and writing

$$
\operatorname{PD}\left(\Delta_{s}\right)=\sum_{p, q} Q_{p, q}^{V} h^{p} \times h^{q}=\sum_{p} h^{p} \times h_{p}
$$

where $Q_{p, q}^{V}$ is the intersection matrix of the Poincaré dual basis, and $\left\{h_{p}\right\}$ is the dual basis with respect to $Q^{V}$. In fact, fix a basis $\left\{\gamma^{i}\right\}$ of $H_{*}(V)$, and let $\left\{\gamma_{i}\right\}$ be the dual basis of $H_{*}(V)$ with respect to the intersection form. Then the $\left\{h^{p}\right\}$ can be taken to be the basis $\left\{C_{s, \gamma^{I}}^{*}=C_{s, \gamma^{1}}^{*} \otimes \cdots \otimes C_{s_{\ell}, \gamma^{\ell}}^{*}\right\}$ of Example 2.4. The convolution then has the more explicit form

$$
(\kappa \otimes h) *\left(\kappa^{\prime} \otimes h^{\prime}\right)=\sum_{B, C, s} \frac{|s|}{\ell(s) !}\left(\xi_{\ell(s)}\right)_{*}\left(\kappa \otimes \kappa^{\prime}\right) \sum_{I} C_{s, \gamma^{I}}^{*}(h) C_{s, \gamma_{I}}^{*}\left(h^{\prime}\right) t_{B+C} \lambda^{2 \ell(s)} .
$$


Finally, we can also include the $\psi$-classes (1.6) to obtain a sum formula for the numbers

$$
T W_{\chi, A, D, X}(\kappa, \alpha)
$$

in terms of the relative invariants (also numbers) $T W_{\chi, A, D, X}^{V}\left(\kappa, \alpha ; C_{s, \gamma}^{*}\right)$. For this, we will take $\kappa=1$ and pair each $\psi$ class with an $\alpha$. This case is usually written in terms of the classes $\tau_{d}(\alpha)=\psi_{i}^{d} \cup e v_{i}^{*}(\alpha)$, so we adopt the notation

$$
T W_{\chi, A, X}^{V}\left(\tau_{D}(\alpha) ; s, \gamma\right):=T W_{\chi, A, D, X}^{V}\left(1, \alpha ; C_{s, \gamma}^{*}\right)
$$

Using dual bases $\left\{\gamma^{I}, \gamma_{I}\right\}$ as above, we let $T W_{\chi, A}\left(s_{1}, \gamma^{I} ; s_{2}, \gamma_{J}\right)$ denote the relative invariant of $\mathbb{F}$ satisfying the contact constraints $C_{s_{1}, \gamma^{I}}^{*}$ along $\bar{V}$ and $C_{s_{2}, \gamma_{J}}^{*}$ along $V$. Then the inverse $S$-matrix is the alternating series

$$
\begin{aligned}
& \text { (4.1) } \begin{array}{l}
S_{\chi, A}^{\bar{V}, V}\left(s_{1}, \gamma_{I} ; s_{2}, \gamma^{J}\right)= \\
\mathbb{I}\left(s_{1}, \gamma_{I} ; s_{2}, \gamma^{J}\right)-T W_{\chi, A}^{\bar{V}, V}\left(s_{1}, \gamma_{I} ; s_{2}, \gamma^{J}\right)+ \\
\sum_{\substack{A_{1}+A_{2}=A \\
\chi_{1}+\chi_{2}-2 \ell\left(s_{3}\right)=\chi}} \sum_{s_{3}, I_{3}} \frac{\left|s_{3}\right|}{\ell\left(s_{3}\right) !} T W_{\chi_{1}, A_{1}}^{\bar{V}, V}\left(s_{1}, \gamma_{I} ; s_{3}, \gamma^{I_{3}}\right) T W_{\chi_{2}, A_{2}}^{\bar{V}, V}\left(s_{3}, \gamma_{I_{3}} ; s_{2}, \gamma^{J}\right)-\ldots
\end{array}
\end{aligned}
$$

Theorem 4.3. Suppose there are no rim tori in $(X, V)$ and $(Y, \bar{V})$ and that the constraint $\alpha$ separates as in (3.4). Then the number

$$
T W_{\chi, A, Z}\left(\tau_{D}(\alpha)\right)
$$

is given by

$$
\begin{array}{r}
\sum \frac{\left|s_{1}\right|\left|s_{2}\right|}{\ell\left(s_{1}\right) ! \ell\left(s_{2}\right) !} T W_{\chi_{1}, A_{1}, X}^{V}\left(\tau_{D}\left(\alpha_{X}\right) ; s_{1}, \gamma^{I}\right) S_{\chi_{0}, A_{0}}^{\bar{V}, V}\left(s_{1}, \gamma_{I} ; s_{2}, \gamma^{J}\right) \\
T W_{\chi_{2}, A_{2}, Y}^{\bar{V}}\left(s_{2}, \gamma_{J} ; \quad \tau_{D}\left(\alpha_{Y}\right)\right)
\end{array}
$$

where the sum is over all (a) decompositions $A=A_{1}+A_{0}+A_{2}$ and $\chi=\chi_{1}+\chi_{0}+$ $\chi_{2}-2 \ell\left(s_{1}\right)-2 \ell\left(s_{2}\right)$, (b) pairs of sequences $s_{1}, s_{2} \in \mathcal{S}$ of degree $\operatorname{deg} s_{i}=A_{i} \cdot V$, and (c) pairs of multiindices $I, J$ enumerating bases $\left\{\gamma^{I}, \gamma_{I}\right\}$ of $H^{*}\left(V^{\ell\left(s_{i}\right)}\right)$ dual by the intersection form.

In the case where $T W_{V, \bar{V}}(1,1)=\mathbb{I}$ only the leading term of (4.1) is present, and this sum formula simplifies to

$$
\sum_{\substack{A=A_{1}+A_{2} \\ \chi_{1}+\chi_{2}-2 \ell(s)=\chi}} \sum_{s, I} \frac{|s|}{l(s) !} T W_{\chi_{1}, A_{1}, X}^{V}\left(\tau_{D_{X}}\left(\alpha_{X}\right) ; s, \gamma^{I}\right) T W_{\chi_{2}, A_{2}, Y}^{V}\left(s, \gamma_{I} ; \tau_{D_{Y}}\left(\alpha_{Y}\right)\right) .
$$




\section{Applications}

This section presents two consequences of Theorem 4.1: the Caporaso-Harris recursion formula for the number of curves in $\mathbb{P}^{2}$, and the formula (related to the "Yau-Zaslow conjecture") for the number of curves in the rational elliptic surface. While these formulas are both known, the proofs outlined here are considerably easier and more transparent than the existing ones.

We begin by examining the relative invariants for the pair $\left(\mathbb{P}^{2}, L\right)$ (the manifold $\hat{X}$ of section 2 is then the 4-ball, and in that sense we are considering the relative invariant of $\left.\left(B^{4}, S^{3}\right)\right)$. This pair can be written as a symplectic sum

$$
\left(\mathbb{P}^{2}, L\right) \underset{L=E}{\#}\left(\mathbb{F}_{1}, E, L\right)=\left(\mathbb{P}^{2}, L\right)
$$

where $\left(\mathbb{F}_{1}, E, L\right)$ is the ruled surface with euler class one with its zero section $L$ and its infinity section $E$. We will obtain a recursive formula for the relative invariants of $\left(\mathbb{P}^{2}, L\right)$ by moving one point constraint $p$ to the $\mathbb{F}$ side, and applying the sum formula.

Following $[\mathrm{CH}]$, let $N^{d, \delta}(\alpha, \beta)$ be the number of degree $d$ curves in $\mathbb{P}^{2}$ with $\delta$ double points with a contact with $L$ of order $k$ at $\alpha_{k}$ fixed points, and at $\beta_{k}$ moving points, and passing through the appropriate number of fixed generic points off $L$. Similarly, let $N_{\mathbb{F}}^{a, b, \delta}\left(\alpha^{\prime}, \beta^{\prime} ; p ; \alpha, \beta\right)$ be the number of curves in $\mathbb{F}$ representing $a L+b F$ that have contact described by $\left(\alpha^{\prime}, \beta^{\prime}\right)$ along $E,(\alpha, \beta)$ along $L$, and pass through $p \in \mathbb{F}$. By elaborating on Example 2.4, one can relate these numbers $N^{d, \delta}$ to the relative $T W$ invariants. Theorem 4.1 then becomes

$$
N^{d, \delta}(\alpha, \beta)=\sum\left|\alpha^{\prime}\right|\left|\beta^{\prime}\right| N^{d^{\prime}, \delta^{\prime}}\left(\alpha^{\prime}, \beta^{\prime}\right) \cdot N_{\mathbb{F}}^{d-d^{\prime}, d, \delta^{\prime \prime}}\left(\beta^{\prime}, \alpha^{\prime} ; p ; \alpha, \beta\right),
$$

(the $S$-matrix is the identity by Example 3.4). Furthermore, a dimension count shows that there are exactly two types of curves that contribute to the last term in (5.1): either (i) several rational covers of the fiber, one of them passing through the point $p$, or (ii) several rational covers of the fiber and a rational curve in the class $L+a F$ passing through $p$ and having all contact points with $E$ and $L$ fixed. In each situation there is a unique such curve. In the case (i) nothing changes, except that a contact of some order $k$ at a moving point becomes a contact of order $k$ at the fixed point $p_{0}$ where the fiber through $p$ hits $E$; in case (ii) the degree of the curve drops by 1 , and the curve loses some of the contact conditions at fixed points and gains some at moving points, so $\alpha \geq \alpha^{\prime}$ and $\beta^{\prime} \geq \beta$. Thus the product in (5.1) becomes a sum of two terms. The resulting formula has the form

$$
\begin{aligned}
& N^{d, \delta}(\alpha, \beta)= \\
& \sum k N^{d, \delta^{\prime}}\left(\alpha-\varepsilon_{k}, \beta+\varepsilon_{k}\right)+\sum\left|\beta^{\prime}-\beta\right| \cdot\left(\begin{array}{c}
\alpha \\
\alpha^{\prime}
\end{array}\right)\left(\begin{array}{c}
\beta^{\prime} \\
b
\end{array}\right) \cdot N^{d-1, \delta^{\prime}}\left(\alpha^{\prime}, \beta^{\prime}\right),
\end{aligned}
$$

and is exactly the formula of Caporaso-Harris ([CH] Theorem 1.1). 
The same method, applied to the sum $\mathbb{F}_{n} \# \mathbb{F}_{n}=\mathbb{F}_{n}$ yields the recursion formula of Vakil [V].

We next consider the invariants of the rational elliptic surface $E$. Let $f$ and $s$ denote, respectively, the homology class of the fiber and the section of the elliptic fibration $E \rightarrow \mathbb{P}^{1}$. We will consider the invariants $G W_{g, s+d f}$, which count the number of connected genus $g$ curves in the class $s+d f$ through $g$ generic points. These define power series

$$
F_{g}(t)=\sum_{d \geq 0} G W_{g, s+d f}\left(p^{g}\right) t^{d} t_{s}
$$

where $t=t_{f}$. Recently, Göttsche and Pandharipande [GP] obtained recursive relations for the coefficients of $F_{0}$, and Bryan-Leung [BL] proved the closed-form expression

$$
F_{0}(t)=t_{s}\left(\prod_{d} \frac{1}{1-t^{d}}\right)^{12}
$$

(this formula was very strongly motivated by the work of Yau-Zaslow [YZ]). We will prove this by relating it to the similar series of elliptic $(g=1)$ invariants

$$
H(t)=\sum_{d \geq 0} G W_{1, s+d f}\left(\tau_{1}\left(f^{*}\right)\right) t^{d} t_{s}
$$

where $f^{*} \in H^{2}(E)$ is the Poincaré dual of the fiber class. The "topological recursion relation" for $g=1$ (TRR) easily implies that

$$
H(t)=\frac{1}{12}\left(t F_{0}^{\prime}-F_{0}\right)+F_{0} G,
$$

where $G=\sum \sigma(d) t^{d}$ is the generating function for $\sigma(d)=\sum_{a \mid d} a$. On the other hand, we can write $E=E \#_{F} T^{2} \times S^{2}$, and compute $H$ by moving the constraint onto the $T^{2} \times S^{2}$ side and using Theorem 4.1. For this situation, the only possible splitting is a genus 0 curve on $E$ side representing $s+d_{1} F$ and a genus 1 curve on the $T^{2} \times S^{2}$ side. But the genus 1 invariant of $T^{2} \times S^{2}$ is computable from TRR, and comes out to be $2(G(t)-1 / 24)$. Therefore,

$$
H(t)=2 F_{0}\left(G-\frac{1}{24}\right)
$$

Equating this with (5.4), we see that $F_{0}$ satisfies the ODE

$$
t F_{0}^{\prime}=12 G F_{0},
$$

with $F_{0}(0)=G W_{0, s} t_{s}=t_{s}$. Integrating and rearranging gives (5.3). 
Finally, by applying Theorem 4.1 to the fiber sum $E \# E=K 3$ and noting that there are no $J$-holomorphic curves in $K 3$ for generic $J$, we find that $F_{g+1}=F_{g} G^{\prime}$. Thus, as in $[\mathrm{BL}]$,

$$
F_{g}(t)=t_{s}\left(G^{\prime}(t)\right)^{g}\left(\prod_{d} \frac{1}{1-t^{d}}\right)^{12} .
$$

\section{References}

[BL] J. Bryan and N.-C. C. Leung, The enumerative geometry of K3 surfaces and modular forms, preprint.

[CH] L. Caporaso and J. Harris, Counting plane curves in any genus, Invent. Math. 131 (1998), 345-392.

[GP] L. Göttsche and R. Pandharipande, The quantum cohomology of blow-ups of $P^{2}$ and enumerative geometry, preprint.

[IP1] E. Ionel and T. Parker, The Gromov invariants of Ruan-Tian and Taubes, Math. Res. Lett. 4, 521-532 (1997).

[IP2] E. Ionel and T. Parker, Gromov-Witten invariants of symplectic sums, in preparation.

[LR] A.-M. Li, Y. Ruan, Symplectic surgery and Gromov-Witten invariants of Calabi-Yau 3-folds I, preprint.

[LT] J. Li and G. Tian, Virtual moduli cycles and Gromov-Witten invariants of general symplectic manifolds, preprint.

[RT] Y. Ruan and G. Tian, Higher genus symplectic invariants and sigma model coupled with gravity, preprint.

[T] C. H. Taubes, Counting pseudo-holomorphic curves in dimension 4, J. Differential Geom. 44 (1996), 818-893.

[V] R. Vakil, Counting curves of any genus on rational ruled surfaces, preprint.

[YZ] S.T. Yau and E. Zaslow, BPS states, string duality, and nodal curves on K3, preprint.

Department of Mathematics, Massachusetts Institute of Technology, Cambridge, MA 02139

E-mail address: ionel@math.mit.edu

Department of Mathematics, Michigan State University, East Lansing, Mi 48824

E-mail address: parker@math.msu.edu 